\section{Congenital nuclear cataracts and uveitis in HIV-transgenic mice}

\begin{abstract}
\end{abstract}
Purpose Patients infected with HIV-1 develop ocular manifestations, some due to opportunistic infections and others attributed to the virus itself. Among the latter are retinal microvasculopathy and uveitis. We have analysed the ocular phenotype in HIVtransgenic mice.

Methods We have studied T26 transgenic mice which bear a gag-pol deleted HIV-1 genome. Transgene RNA was detected by Northern analysis. Ocular pathology was assessed by conventional histology, immunostaining for gp120 envelope protein, and in situ apoptosis detection with endlabelling.

Results Abnormalities of lens epithelial cell development were detected as early as embryonic day 14.5. Histological changes included the malformation of an embryonal lens nucleus and poor closure of the lens suture lines. This resulted in congenital nuclear cataracts, as occur in congenital viral infections in human patients. In the adult animals, lenses were notable for extensive vacuolation, liquefaction, and degeneration of the cortex. Mild iridocyclitis and vitritis were also noted in adult transgenic mice. Immunostaining demonstrated the expression of gp 120 envelope protein within the lens epithelial and fibre cells. Endlabelling with terminal deoxyribonucleotidyl transferase showed increased numbers of apoptotic cells in the adult lens.

Conclusions These findings suggest that one or more HIV-1 proteins are associated with congenital nuclear cataract formation and uveitis in HIV-transgenic mice.

Eye (2002) 16, 177-184. DOI: 10.1038/

sj/EYE/6700101

Keywords: envelope protein; gp 120; HIV-1; nuclear cataract; transgenic mouse; uveitis
MM Mozes ${ }^{1,2}, J L$ Bryant $^{3}$, R Franks ${ }^{4}$, CC Chan ${ }^{5}$ and JB Kopp ${ }^{1}$

Introduction

HIV-1 infected patients are at risk for several intraocular diseases. ${ }^{1}$ The most common complication is retinal microvasculopathy, resulting in retinal haemorrhage and infarcts of the nerve fibre layer, forming cotton-wool spots. ${ }^{2}$ The vasculopathy appears to be due to a direct effect of the virus, independent of opportunistic infection. Indeed, the vascular pathology may be a result of infection of retinal endothelial cells. ${ }^{3}$ Complications due to opportunistic infections include retinitis due to cytomegalovirus, toxoplasmosis and acute retinal necrosis due to herpes simplex or herpes zoster infection. Other ocular disorders in HIV disease include neoplasms, particularly Kaposi's sarcoma, neuro-ophthalmic manifestations, and in some instances uveitis. $^{4-7}$ By contrast, uveitis is relatively common in patients infected with HTLV-I. ${ }^{8,9}$ Uveitis also occurs in rabbits experimentally infected with HTLV-I ${ }^{10}$ and in cats infected with feline leukaemia virus. ${ }^{11,12}$ A preliminary report suggests that lens opacities may also develop in patients infected with HIV-1. ${ }^{13}$

Transgenic mice bearing single or multiple HIV-1 genes have proved useful in defining the tissue-specific pathological role of HIV-1 gene products, independent of immune deficiency or immunosuppression. In this regard, it is notable that cataracts have developed in mice transgenic for HIV-1 as reported previously. Iwakura et al ${ }^{14}$ described a mouse transgenic for a full-length HIV-1 genome, with a deletion in pol, and noted the development of cataracts. p24 Gag protein was detected in the lens fibre cells and the retina, and the authors ascribed the pathogenesis of cataracts to this protein. More recently, cataracts have been described in mice transgenic for the HIV-1 protease under the control of the lens alpha A-crystallin promoter. ${ }^{15,16}$ The authors concluded that proteolysis of lens proteins, presumably induced by the viral protease, was responsible for cataract formation. Most recently, Dickie ${ }^{17}$
${ }^{1}$ Kidney Disease Section Metabolic Diseases Branch National Institute of Diabetes and Digestive and Kidney Disease $\mathrm{NIH}$, Bethesda, MD, USA

2Institute of Pathophysiology Semmelweis University Medical School Budapest, Hungary

${ }^{3}$ Institute of Human Virology

University of Maryland Baltimore, MD, USA

${ }^{4}$ Dept of Molecular Genetics

College of Medicine University of Illinois at Chicago

Chicago, IL, USA

${ }^{5}$ Laboratory of Immunology National Eye Institute $\mathrm{NIH}$, Bethesda, MD, USA

Correspondence:

J Kopp, MD

Building 10, Room 3N116

National Institutes of Health

Bethesda

MD 20892-1268, USA

Tel: +1 (301) 5943403

Fax: + 1 (301) 4020014

E-mail: jbkopp@nih.gov

Received: 13 October 1999 Accepted in revised form: 22 June 2001 
reported on cataracts occurring in five transgenic lines of mice derived from four constructs: intact HIV-1 proviral DNA under the control of the HIV-1 long terminal repeat (LTR) or a chimeric HIV-1/MMTV LTR, a gag-pol deleted genome and the Nef gene. This author showed that cataracts occurred in mice which expressed HIV-1 RNA in the eye and Nef protein within the lens. He noted lens opacity and architectural disruption in T26 mice bearing the gag-pol deleted genome. Whereas normal lens showed an equatorial distribution of anterior epithelial cell layer and parallel lens fibres, in the transgenic lens the epithelial cells were displaced peripherally, and both the lens fibres had lost orientation. Increased apoptosis was present in lens tissue. Dickie concluded that Nef perturbed lens cell terminal differentiation and that this resulted in cataract formation.

The above reports have implicated different HIV-1 proteins in the pathogenesis of cataracts in transgenic mice. We therefore felt that it would be productive to characterise in greater detail the histopathology of the cataracts and other ocular pathology occurring in the T26 mice with a gag-pol deleted HIV-1 genome, and to provide a complete description of the lens abnormalities and associated ocular pathology.

\section{Materials and methods}

\section{Transgene}

The transgenic line T26 has been described previously. ${ }^{18}$ This line contains a genome termed d1443 and represents a gag-pol deleted genome (Figure 1) derived from pNL43 under the control of the viral LTR and injected into FVB/N oocytes. Eight founder mice were obtained and seven transgenic lines were established; only one line manifested cataracts. Transgene RNA, assessed by Northern analysis, is expressed abundantly in muscle and skin and to a lesser extent in kidney, lymphoid tissue, intestine and eye. Other phenotypes included focal glomerulosclerosis, ${ }^{19}$ cutaneous papillomas ${ }^{20}$ and, in the homozygote, wasting and early death. ${ }^{21}$ Transgene status was determined by Southern blotting, as described previously. ${ }^{19}$ Embryonic stages were determined by assigning the day of the vaginal plug as 0.5. Mice were cared for according to NIH animal care guidelines and were killed by carbon dioxide inhalation followed by cervical dislocation. Care adhered to the ARVO Statement for the Use of Animals in Ophthalmic and Vision Research.

\section{Northern analysis}

Total RNA was prepared from eyes by homogenising in guanidine and centrifuging through a caesium chloride cushion. Northern analysis was performed using total RNA. After transfer to a Nytran membrane (Schleicher and Schuell, Keene, NH, USA) the blot was probed with a labelled cDNA (GM91, including the HIV-1 nef gene, deposited by John Rossi with the AIDS Reference Reagent and Repository Program (ARRRP), Rockville, MD, USA).

HIV-1 Open Reading Frames

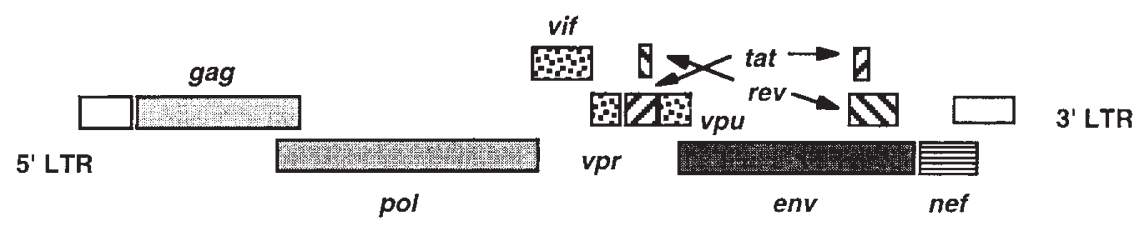

gag/pol deleted construct ( $p N L 4-3:$ d1443)

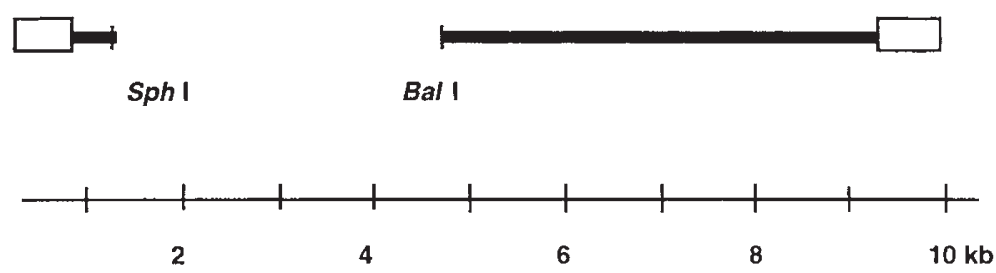

Figure 1 HIV-1 genome. The open reading frames of HIV-1 are shown above. The gag, pol and env genes represent the minimal complement of genes present in all retroviruses, together with the long terminal repeats (LTR). The regulatory and accessory genes of HIV include tat, rev, nef, vif, vpr and vpu. The gag-pol deleted HIV-1 genome, which was derived from the infectious clone pNL43 and is present in the T26 mice studied here, is shown below. 


\section{Histology}

Embryonic eyes were removed and fixed in $10 \%$ neutral buffered formalin and embedded in glycol methacrylate. Newborn and adult eyes were removed, fixed in $4 \%$ glutaraldehyde in phosphate buffer for $30 \mathrm{~min}$ at $4^{\circ} \mathrm{C}$ and then fixed in $10 \%$ neutral buffered formalin overnight at room temperature. Paraffin sections $(4 \mu \mathrm{m})$ and glycol methacrylate plastic sections $(2 \mu \mathrm{m})$ were made through the pupillary-optic nerve plane and stained with haematoxylin and eosin.

\section{Immunohistochemistry}

Eyes were fixed in methanol Carnoy's solution (60\% methanol, 30\% chloroform, 10\% glacial acetic acid) overnight at room temperature. After paraffin embedding, $4 \mu \mathrm{m}$ sections were cut. Histochemical detection of HIV-1 envelope protein was performed exactly as previously described, ${ }^{20}$ using a polyclonal sheep anti-gp120 antiserum (developed by M Phelan and obtained from ARRRP). This antiserum or its control, normal sheep serum, were diluted 1:2000 and applied to the sections for $1 \mathrm{~h}$ at $37^{\circ} \mathrm{C}$. Bound antibody was detected using a commercial streptavidinperoxidase kit (Zymed, Burlingame, CA, USA).

\section{Apoptosis detection}

Apoptotic cells were detected with the TUNEL (terminal deoxynucleotidyl transferase end-labelling) technique, using a TACS BlueLabel Detection kit (Trevigen, Gaithersburg, MD, USA) according to the manufacturer's protocol. This approach labels free 3'hydroxyl DNA ends, which are numerically increased in cells undergoing apoptosis. Briefly, formalin-fixed, paraffin-embedded tissue sections were deparaffinised and rehydrated. The slides were then treated with proteinase $\mathrm{K}$ ( $1 \mathrm{mg} / \mathrm{ml}$ for $3 \mathrm{~min}$ ) to increase tissue permeability, and the slides exposed to $2 \% \mathrm{H}_{2} \mathrm{O}_{2}$ for $5 \mathrm{~min}$ to quench endogenous peroxidases. The tissue sections were in situ labelled with a biotin-labelled dNTP mix and terminal deoxynucleotidyl transferase (TdT) in the presence of $\mathrm{MnCl}_{2}$ while incubated at $37^{\circ} \mathrm{C}$. The reaction was terminated by incubation with the stop buffer as supplied by the manufacturer. Streptavidin-horseradish peroxidase conjugates were added on the tissue, followed by a substrate yielding a blue colour. The sections were then counterstained with Red Counterstain C, mounted and photographed.

\section{Results}

\section{Transgene expression}

T26 mice consistently showed transgene expression in the eye. Thus in a T26 mouse killed at 11 days of age, transgene RNA was present in skin, eye, thymus, skeletal muscle and colon (Figure 2). In these tissues, $2 \mathrm{~kb}$ multiply-spliced and $4 \mathrm{~kb}$ single-spliced RNA predominated; $7 \mathrm{~kb}$ unspliced transgene RNA was also detected.

\section{Ocular clinical presentation}

The T26 transgenic line has been maintained for over 8 years and has demonstrated a stable ocular phenotype during that time. Cataracts were present by embryonic day 14.5 (E14.5) and were present in $100 \%$ of both heterozygotes and homozgyotes. Both genotypes also manifested mild microphthalmia. Cataracts were consistently present when the T26 line was bred on to other genetic backgrounds, including C57/Bl6. In addition, cataracts were present when the T26 mouse was bred onto the FVB/N athymic nude mouse background.

\section{Histology}

Cataract formation in the HIV-transgenic mice was identified as early as E14.5. This was characterised by the malformation or impaired formation of fetal lens

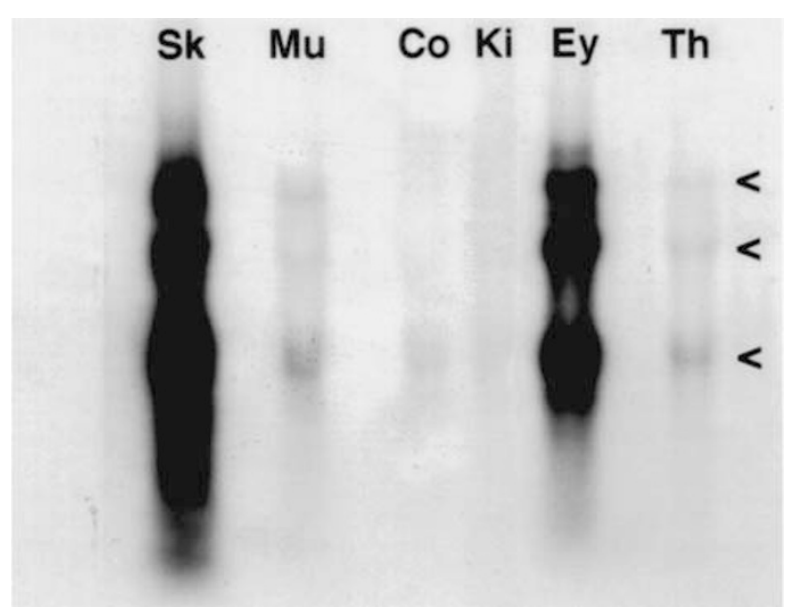

Figure 2 Northern analysis of transgene expression. Total RNA was prepared from the following organs of an 11-day-old T26 mouse and was electrophoresed in the following amounts: skin (25 $\mu \mathrm{g}, \mathrm{SK})$, skeletal muscle $(25 \mu \mathrm{g}, \mathrm{Mu})$, colon $(25 \mu \mathrm{g}, \mathrm{Co})$, kidney ( $25 \mu \mathrm{g}, \mathrm{Ki})$, eye (15 $\mu \mathrm{g}$, Ey) and thymus (13 $\mu \mathrm{g}$, Th). An HIV-1 cDNA was used to demonstrate the expression of HIVtransgene RNA, with sizes of $7 \mathrm{~kb}, 4 \mathrm{~kb}$ and $2 \mathrm{~kb}$ RNA (indicated by arrows). Specific viral RNAs are particularly abundant in skin and eye. 
nucleus (Figure 3b). Cataractous changes were extensive, with abnormal formation of fetal nucleus and poor closure of lens suture lines (Figure 3d). Lenses with relatively normal contours showed only minimal cortical degeneration, while those that were more spherophakic exhibited cortical cataractous changes of vacuoles and globulin formation, liquefaction, and fragmentation (Figure 3f, h). Mild iridocyclitis and vitritis were also noted in adult transgenic mice, with infiltration of mononuclear cells consistent with lymphocytes and monocytes (Figure 4a, b). In contrast, wild-type mice demonstrated normal lens architecture, forming acellular homogeneous lens nuclei and cortex in both the fetus and adult (Figure $3 e, g$ ). The remainder of the eye was normal except for congenital retinal degeneration, which is characteristic of the albino FVB/N mouse (data not shown).

\section{Immunolocalisation of HIV protein}

Using immunohistochemistry and antiserum to gp120 HIV-1 envelope protein, we localised gp 120 protein to the cytoplasm of lens epithelial fibre cells in newborn transgenic mouse lens, where it is detected as deposits of intense red stain (Figure $4 c, d$ ). The remainder of the eye did not show specific staining for gp 120. The lens of wild-type mice, exposed to specific gp 120 antiserum, showed no staining (Figure 4e)

\section{Apoptosis}

In adult wild-type mice, epithelial and fibre cells at the periphery of the lens rarely labelled with the TUNEL method, indicating that few or no cells were undergoing apoptotic cell death (Figure 4g). By contrast, the lens from an adult T26 mouse showed a markedly increased number of apoptotic lens epithelial and lens fibre cells, especially in regions of the lens affected by cataractous changes (Figure 4f). In the absence of terminal deoxynucleotidyl transferase enzyme, there was no tissue staining (Figure $4 \mathrm{~h}$ ).

\section{Discussion}

HIV-transgenic mice provide a useful model to dissect the pathogenesis of congenital cataracts associated with viral infections occurring during fetal development. In normal lens development, karyolysis leads to the disappearance of nuclei in all but the outer cortical fibres of the lens. In certain viral infections, there is a delay or absence of normal karyolysis and the abnormal survival of the lens epithelial and fibre cells is associated with the formation of congenital nuclear cataracts. The most widely studied example of viralinfection-associated cataract formation occurs following congenital rubella infection. ${ }^{22}$ The rubella virus is thought to integrate into the genomic DNA of lenticular cells, and viral gene expression then interferes with karyolysis. In patients with congenital rubella infection, the lens exhibits spherophakia, with the most marked opacification located centrally. The lens nucleus is sclerotic and the nuclei of the lens epithelial cells are pyknotic. By contrast, the normal lens is free of cell nuclei and appears homogeneous.

The present model shares some important similarities with these observations from human pathology, including increased lens cell number during fetal life and subsequently increased apoptotic cell death. Thus HIV-1 gene expression in the transgenic mouse appears to perturb fetal lens epithelial development and results in the development of the congenital nuclear cataracts in the microphthalmic eye. Abnormalities of lens epithelial cell development appeared as early as E14.5. The lens exhibited a malformed fetal nucleus and poor closure of the lens suture lines. These developmental abnormalities led to congenital nuclear cataracts and indeed the cataracts were macroscopically visible by at least E18.5. In adult animals, there was a range in cataract severity, with the more severely affected mice having lenses with marked vacuolation, liquefaction, fragmentation and apoptosis. Mild iridocyclitis and vitritis were also noted in adult transgenic mice. HIV RNA was abundantly expressed in the eye, and at least one HIV protein, gp120 envelope protein, was present within the lens epithelial cell. We report here several pathological features in this model that have not previously been reported: (1) the cataracts begin in a nuclear location within the lens and subsequently develop as cortical cataracts; (2) uveitis is also present; (3) lens epithelial and fibre cells express HIV gp120 protein; and (4) epithelial and fibre cells exhibit enhanced apoptosis. Taken together, these findings suggest that HIV gene products induce toxicity to the lens epithelial and fibre cells and that this process, initiated during embryogenesis, gives rise to congenital nuclear cataracts.

There are several limitations of the present study.

Figure 3 Histology. (a) In an E14.5 wild-type mouse, anterior cuboidal lens epithelial cells are arranged in a single layer. In the central portion of the lens, primary lens epithelial and fibre cells demonstrate an elongated morphology and uniformly fill the lens vesicle to form the fetal nucleus. (b) In an E14.5 HIVtransgenic mouse, the anterior cuboidal epithelial cells are arranged in multiple layers. Centrally the primary lens fibre cells lack normal elongation and vacuoles are present in the embryonal nucleus. (Continued on facing page) 


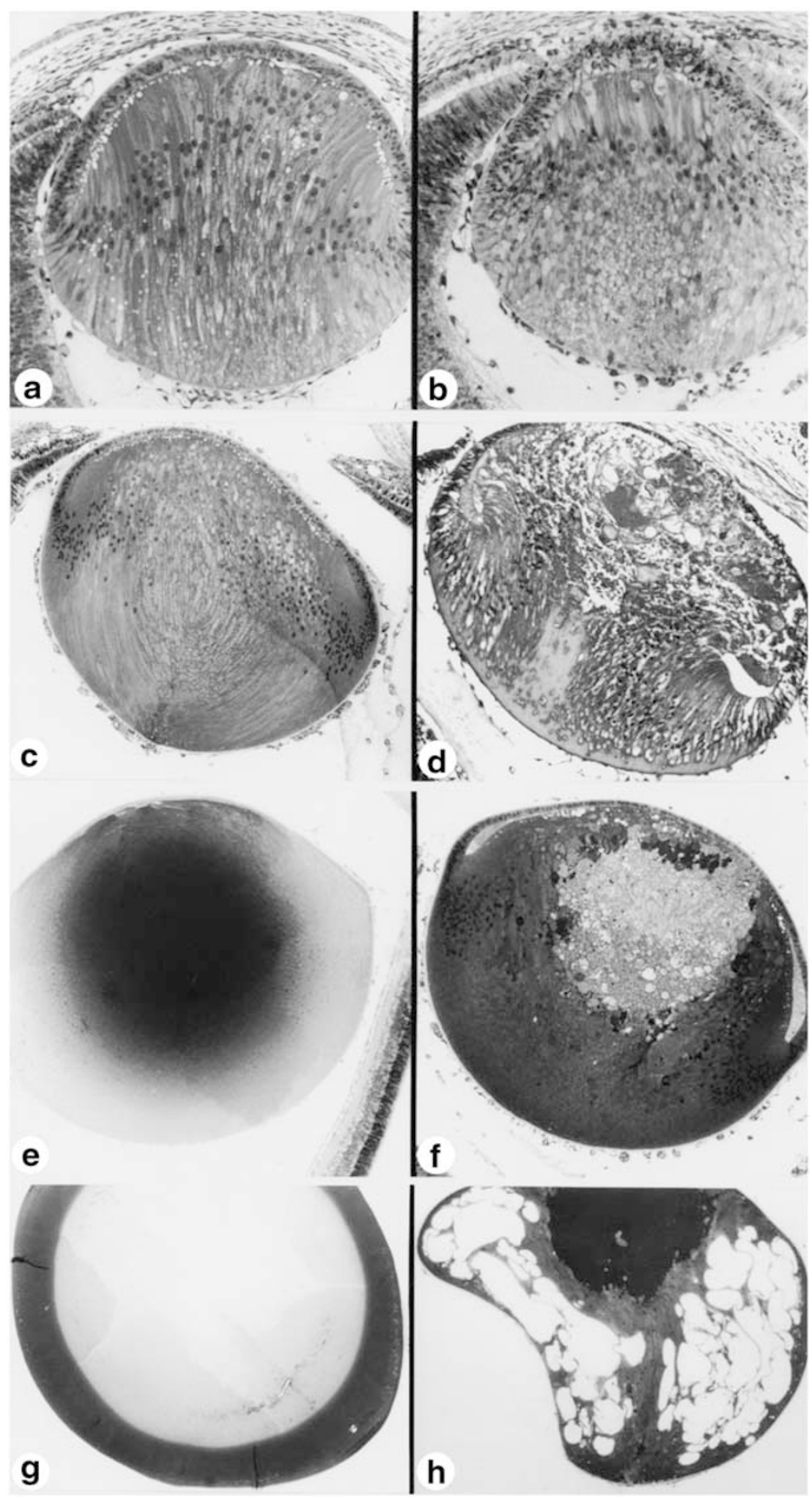

Figure 3 Continued (c) In an E16.5 wild-type mouse, the lens nuclei are homogeneous and lens architecture is normal. (d) In an E16.5 HIV-transgenic mouse, the fetal lens architecture shows progressively more severe disruption, with vacuoles and globulin present in the fetal lens nucleus. (e) In an E18.5 wild-type mouse, the lens is characterised by acellular and homogeneous lens nucleus and cortex, with a single layer of anterior and peripheral lens epithelial cells. There is a nuclear bow at the lens equator. (f) In a E18.5 HIV-transgenic mouse, a lens with a spherical shape contains a well-demarcated, sclerotic lens nucleus. The lens fibre cell nuclei show an abnormal persistence beyond the nuclear bow and posterior epithelial cell migration, pyknosis and karyorhexis. The anterior cortex shows marked degeneration and liquefaction, while the anterior lens epithelial cells are relatively normal at this stage. (g) In an adult wild-type mouse, the lens has a uniformly normal nucleus and cortex. (h) In an adult HIV transgenic mouse, there are both nuclear cataracts (appearing as darkly stained central matter) and cortical cataracts (appearing as vacuolar areas at the periphery of the lens). All sections were stained with haematoxylin and eosin and photographed at $\times 62.5$. 

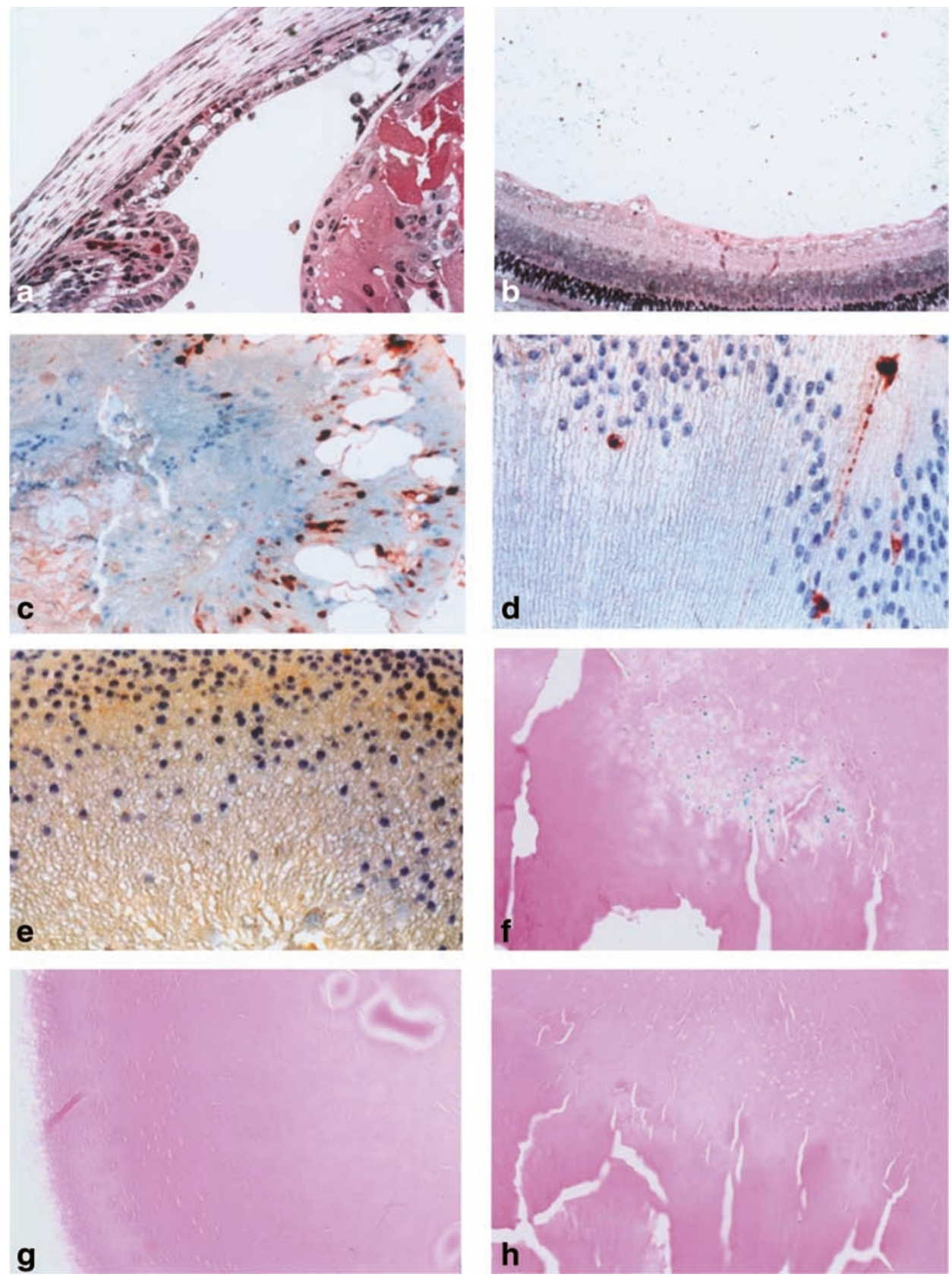

Figure 4 Histology, immunostaining for gp120 protein and TdT-mediated detection of apoptosis. Haematoxylin-stained sections of HIV-transgenic mouse eyes aged 8 weeks shows mononuclear cells and neutrophils (arrowheads) located in the posterior chamber, adjacent to the cataractous lens (a), while similarly stained sections from a transgenic mouse aged 12 weeks show inflammatory cells (arrowheads) present in the vitreous (b). Sections from newborn T26 mouse eyes (c, d) or littermate normal mouse eyes (e) were incubated with polyclonal anti-gp120 antiserum, detected with immunoperoxidase technique. gp120 protein is expressed within lens epithelial and fibre cell cytoplasm, as shown by a dense red cell-associated deposit which is entirely lacking from wild-type mouse sections. Sections of adult T26 mouse eye (f, h) and wild-type mouse eye (g) were processed for terminal deoxynucleotidyl transferase end labelling to detect apoptotic cells in the lens. As a control, T26 mouse eye was processed in an identical fashion but terminal deoxynucleotidyl transferase was omitted $(h)$. Increased numbers of apoptotic cells were present in transgenic adult mouse eye within the lens cortex (f) compared with wild-type mouse eye (g), where no apoptotic cells are shown. Magnification: (a) $\times 40$, (b) $\times 20$, (c) $\times 62.5, \mathrm{~d}-\mathrm{e} \times 100, \mathrm{f}-\mathrm{h} \times 20$. 
First, since only one of seven transgenic lines developed cataracts, we cannot formally exclude the possibility that the phenotype derives from interruption by the transgene of a gene essential for normal ocular development. The remaining lines are not presently available for further study. However, the observation that other HIV-1 transgenic mice have developed cataracts and that this phenotype is not commonly associated with transgenic animals argues for the role of HIV-1 proteins in inducing the ocular disease reported here. Second, the transgene in the present study includes open reading frames for multiple HIV-1 proteins, including envelope protein and the accessory proteins Tat, Rev, Nef, Vif, Vpr and Vpu. While we detected gp 120 envelope protein in the lens, this finding does not establish which HIV-1 gene product or products is responsible for ocular disease. Determining this would require other models, particularly including mice transgenic for single viral genes. HIV-1 has been reported to infect retinal cells in culture $^{23}$ and in vivo. ${ }^{24}$

Comparisons between the phenotype of HIVtransgenic mice and the syndromes seen in patients with HIV-1 infection must be made with caution. HIV1 has been reported to infect retinal cells in culture ${ }^{23}$ and in vivo. ${ }^{24}$ In the mouse, the lens epithelial and fibre cells are permissive for transgene expression under the control of the HIV-1 LTR. ${ }^{25}$ Our studies confirm that lens epithelial and fibre cells express HIV-1 proteins. It is important to note that ectopic protein expression in the lens is insufficient to produce cataracts or other pathology in all cases, as shown by the normal histology present in the eyes of mice expressing HIV-1 Tat under the control of the alpha A-crystallin promoter. ${ }^{26}$ It is important to note that cataracts have not been explicitly associated with HIV-1 infection in human patients. A preliminary report does note an increased prevalence of lens opacities in adult patients with HIV-1 infection, but these findings have not been fully defined. ${ }^{13}$ We observed a low-grade uveitis in HIV-transgenic mice. HIV-1 infection is rarely associated with uveitis, but both anterior and posterior uveitis have been reported. ${ }^{6,7}$ Recently, immune recovery uveitis has been recognised in AIDS patients who have received highly active anti-retroviral therapy. ${ }^{27}$ This syndrome includes transient ocular inflammation, including vitritis and anterior uveitis. Immune recovery uveitis is thought to result from restoration of immune function and consequent immunological recognition of opportunistic infections of the ocular tissues. Our data suggest the alternative possibility that HIV-1 viral products contribute to the process of uveitis and cataract formation.

The HIV-1 gene responsible for the ocular pathology described here cannot be identified with certainty. It has been suggested the HIV-1 protease gene, when expressed in transgenic mice, induces cataracts, ${ }^{28}$ but the HIV-1 protease gene is absent from the T26 transgenic mice used in the present study. We have localised gp120 to lens epithelial and fibre cells in T26 mice. Earlier studies with a variety of HIV-transgenic mice have localised p24 Gag protein, HIV protease and Nef protein to these cells. Dickie ${ }^{17}$ attributed cataract formation in the HIV-transgenic mice reported on here to the presence of Nef protein within the lens. In mice transgenic for the HIV-1 nef gene alone, this author described a small, central lens opacity which was most prominent in the lens of newborn mice and which faded with age, leaving only a mild persistent opacity. Uveitis was absent from this model. By contrast, the cataracts in the T26 mouse progressively enlarge and increase in opacity with age, and uveitis is also present. These differences suggest the possibility that other HIV-1 proteins, such as gp120, may modify the phenotype. Further studies of cataract formation in transgenic models may extend our understanding of congenital and acquired cataracts in human patients.

\section{Acknowledgements}

The authors wish to acknowledge the technical assistance of Charles Wohlenberg with animal care, Dawn Matteson with TUNEL assay, and Lisa Miller with preparation of the photographic figures, and the critical review of the manuscript by Dr Lisa Hensley.

\section{References}

1 Ugen KE, McCallus DE, Von Feldt JM, Williams WV, Greene MI, Weiner DB. Ocular tissue involvement in HIV infection: immunological and pathological aspects. Immunol Res 1992; 11: 141-153.

2 Geier SA, Schielke E, Klauss V, Muller A, Einhaupl KM, Goebel FD et al. Retinal microvasculopathy and reduced cerebral blood flow in patients with acquired immunodeficiency syndrome. Am J Ophthalmol 1992; 113: 100-101.

3 Pomerantz RJ, Kuritzkes DR, de la Monte SM, Rota TR, Baker AS, Albert D et al. Infection of the retina by human immunodeficiency virus type I. N Engl J Med 1987; 317: 1643-1647.

4 Farrell PL, Heinemann MH, Roberts CW, Polsky B, Gold JW, Mamelok A. Response of human immunodeficiency virus-associated uveitis to zidovudine. Am J Ophthalmol 1998; 106: 7-10.

5 Geny C, Binaghi M, Lionnet F, Deforges L, Defer G, Schaeffer A. Uveomyelitis associated with HIV infection. AIDS 1992; 6: 1220-1221.

6 McCluskey PJ, Wakefield D. Posterior uveitis in the acquired immunodeficiency syndrome. Int Ophthalmol Clin 1995; 35: 1-14.

7 Rosberger DF, Heinemann MH, Friedberg DN, Holland 
GN. Uveitis associated with human immunodeficiency virus infection. Am J Ophthalmol 1998; 125: 301-305.

8 Nakao K, Ohba N, Isashiki M, Isashiki Y, Unoki K, Osame M. Pigmentary retinal degeneration in patients with HTLV-I-associated myelopathy. Jpn J Ophthalmol 1989; 33: 383-391.

9 Mochizuki M, Watanabe T, Yamaguchi K, Yoshimura K, Nakashima S, Shirao M et al. Uveitis associated with human T-cell lymphotropic virus type I. Am J Ophthalmol 1992; 114: 123-129.

10 Taguchi H, Sawada T, Fukushima A, Iwata J, Ohtsuki Y, Ueno $\mathrm{H}$ et al. Bilateral uveitis in a rabbit experimentally infected with human T-lymphotropic virus type I. Lab Invest 1993; 69: 336-339.

11 English RV, Davidson MG, Nasisse MP, Jamieson VE, Lappin MR. Intraocular disease associated with feline immunodeficiency virus infection in cats. J Am Vet Med Assoc 1990; 196: 1116-1119.

12 Callanan JJ, Thompson H, Toth SR, O'Neil B, Lawrence $\mathrm{CE}$, Willett $\mathrm{B}$ et al. Clinical and pathological findings in feline immunodeficiency virus experimental infection. Vet Immunol Immunopathol 1992; 35: 3-13.

13 Gumbel H, Subklew R, Staszewski S, Richter R, Klos K, Ohrloff C. Ocular lens opacities in patients with acquired immunodeficiency syndrome. Int Conf AIDS 1993; 9: 414 (abstract PO-B16-1675).

14 Iwakura Y, Shioda T, Tosu M, Yoshida E, Hayashi M, Nagata $\mathrm{T}$ et al. The induction of cataracts by HIV-1 in transgenic mice. AIDS 1992; 6: 1069-1075.

15 Bettelheim FA, Zeng FF, Bia Y, Tumminia SJ, Russell P. Lens hydration in transgenic mice containing HIV-1 protease linked to the lens alpha A-crystallin promoter. Arch Biochem Biophys 1995; 324: 223-227.

16 Tumminia SJ, Jonak GJ, Focht RJ, Cheng YS, Russell P. Cataractogenesis in transgenic mice containing the HIV-1 protease linked to the lens alpha A-crystallin promoter. $J$ Biol Chem 1996; 271: 425-431.

17 Dickie P. HIV type I nef perturbs eye lens development in transgenic mice. AIDS Res Hum Retrovir 1996; 12: 177189.

18 Dickie P, Felser M, Eckhaus M, Bryant J, Silver J, Marinos $\mathrm{N}$ et al. HIV-associated nephropathy in transgenic mice expressing HIV-1 genes. Virology 1991; 185: 109-119.
19 Kopp JB, Klotman ME, Adler SH, Bruggeman LA, Eckhaus M, Dickie P et al. Progressive glomerulosclerosis and enhanced renal accumulation of basement membrane components in mice transgenic for HIV-1 genes. Proc Nat Acad Sci USA 1992; 89: 1577-1581.

20 Kopp JB, Rooney JF, Wohlenberg C, Marinos NA, Bryant JA, Katz SI et al. Cutaneous disorders and viral gene expression in HIV-1 transgenic mice. AIDS Res Hum Retrovir 1993; 9: 267-275.

21 Santoro TJ, Bryant JL, Pellicoro J, Klotman ME, Kopp JB, Bruggeman LA et al. Growth failure and AIDS-like cachexia syndrome in HIV-1 transgenic mice. Virology 1994; 201: 147-151.

22 Zimmerman LE. The histopathologic basis for ocular manifestations of congenital rubella syndrome. Am J Ophthalmol 1968; 65: 837-862.

23 Dutt K, York D, Kaplan HJ, Semple E, Verly G Srinivasan A. Replication of HIV in human fetal retinal cultures and established pigment epithelial cell lines. Invest Ophthalmol Vis Sci 1989; 30: 1535-1541.

24 Qavi HB, Green MT, Lewis DE, Hollinger FB, Pearson G, Ablashi DV. HIV-1 and HHV-6 antigens and transcripts in retinas of patients with AIDS in the absence of human cytomegalovirus. Invest Ophthalmol Vis Sci 1995; 36: 20402047.

25 Cavard C, Zider A, Vernet M, Bennoun M, Saragosti S, Grimber $\mathrm{G}$ et al. In vivo activation by ultraviolet rays of the HIV-1 long terminal repeat. J Clin Invest 1990; 86: 1369-1374.

26 Khillan JS, Deen KC, Yu S, Sweet RW, Rosenberg M, Westphal H. Gene transactivation mediated by the TAT gene of HIV in transgenic mice. Nucleic Acids Res 1988; 16: $1423-1429$.

27 Karavellas MP, Lowder CY, Macdonald C, Avila CP Jr, Freeman WR. Immune recovery vitritis associated with inactive cytomegalovirus retinitis: a new syndrome. Arch Ophthalmol 1998; 116: 169-175.

28 Mitton KP, Kamiya T, Tumminia SJ, Russell P. Cysteine protease activated by expression of HIV-1 protease in transgenic mice: MIP26 (aquaporin-0) cleavage and cataract formation in vivo and ex vivo. J Biol Chem 1996; 13: 31803-31806. 